\title{
Ecological and Social Factors Constrain Spatial and Temporal Opportunities for Mating in a Migratory Songbird
}

\author{
Sara A. Kaiser, ${ }^{1, \star}$ Benjamin B. Risk, ${ }^{2, \dagger}$ T. Scott Sillett, ${ }^{3}$ and Michael S. Webster ${ }^{1}$ \\ 1. Department of Neurobiology and Behavior, Cornell University, Ithaca, New York 14853; and Macaulay Library, Cornell Lab of \\ Ornithology, Ithaca, New York 14850; 2. Department of Statistical Science, Cornell University, Ithaca, New York 14853; 3. Migratory Bird \\ Center, Smithsonian Conservation Biology Institute, National Zoological Park, Washington, DC 20013 \\ Submitted November 9, 2015; Accepted October 13, 2016; Electronically published February 1, 2017 \\ Online enhancements: appendix, R code. Dryad data: http://dx.doi.org/10.5061/dryad.ck0ts.
}

AвSTRACT: Many studies of sexual selection assume that individuals have equal mating opportunities and that differences in mating success result from variation in sexual traits. However, the inability of sexual traits to explain variation in male mating success suggests that other factors moderate the strength of sexual selection. Extrapair paternity is common in vertebrates and can contribute to variation in mating success and thus serves as a model for understanding the operation of sexual selection. We developed a spatially explicit, multifactor model of all possible female-male pairings to test the hypothesis that ecological (food availability) and social (breeding density, breeding distance, and the social mate's nest stage) factors influence an individual's opportunity for extrapair paternity in a socially monogamous bird, the black-throated blue warbler, Setophaga caerulescens. A male's probability of siring extrapair young decreased with increasing distance to females, breeding density, and food availability. Males on food-poor territories were more likely to sire extrapair young, and these offspring were produced farther from the male's territory relative to males on food-abundant territories. Moreover, males sired extrapair young mostly during their social mates' incubation stage, especially males on food-abundant territories. This study demonstrates how ecological and social conditions constrain the spatial and temporal opportunities for extrapair paternity that affect variation in mating success and the strength of sexual selection in socially monogamous species.

Keywords: breeding density, extrapair paternity, food availability, reproductive synchrony, sexual network, sexual selection.

* Corresponding author. Present address: Center for Conservation Genomics, Smithsonian Conservation Biology Institute, National Zoological Park, Washington, DC 20013; e-mail: kaisers@si.edu.

$\dagger$ Present address: Statistical and Applied Mathematical Sciences Institute, Durham, North Carolina 27709; and Department of Biostatistics, University of North Carolina, Chapel Hill, North Carolina 27599.

ORCIDs: Kaiser, http://orcid.org/0000-0002-6464-3238; Risk, http://orcid.org /0000-0003-1090-0777; Sillett, http://orcid.org/0000-0002-7486-0076; Webster, http://orcid.org/0000-0001-7585-4578.

Am. Nat. 2017. Vol. 189, pp. 283-296. (C) 2017 by The University of Chicago. 0003-0147/2017/18903-56642\$15.00. All rights reserved.

DOI: $10.1086 / 690203$

\section{Introduction}

Sexual selection is the primary evolutionary force shaping sexual traits and is driven by competition for access to mates (Andersson 1994). The opportunity for sexual selection to operate is determined by variance in mating success. When most of that variance is explained by differences in sexual traits (e.g., ornaments, coloration, songs, and courtship displays), the strength of sexual selection will be near its theoretical maximum (Arnold and Wade 1984; Jones 2009). However, many empirical studies examining associations between sexual traits and mating success have found weak or no selection (Westneat 2006; Akçay and Roughgarden 2007; Soulsbury 2010; Hsu et al. 2015), suggesting that mating success is associated with other unmeasured traits or that the strength of sexual selection is moderated by other factors. The nature of those factors on sexual selection remains unclear.

Ecological factors might have significant but largely overlooked effects on the distribution of mating success among individuals, thereby moderating the strength of sexual selection (McDonald et al. 2013). Many studies of sexual selection implicitly assume that all individuals in a population have equal mating opportunities (Andersson and Simmons 2006). This expectation has been challenged by recent evidence that the strength of sexual selection within populations can be highly variable over space and time (Cornwallis and Uller 2010; Miller and Svensson 2014) and can fluctuate with ecological conditions, such as rainfall (Robinson et al. 2012), food availability (Janicke et al. 2015), and population density (Ryder et al. 2012; Taff et al. 2013). These patterns suggest that ecological factors generate unequal spatial and temporal opportunities for mating, irrespective of male phenotypes, leading to variation in the strength of sexual selection across ecological conditions. Therefore, to understand what drives variation in the strength of sexual selection, we need to first evaluate the fundamental assumption that individuals have equal mating opportunities and 
examine how ecological factors affect variation in mating success.

An important source of variation in mating success in many vertebrate populations is extrapair paternity - when males and females mate outside of social pair bonds (fishes [Avise et al. 2002]; birds [Griffith et al. 2002]; reptiles [Uller and Olsson 2008]; mammals [Cohas and Allainé 2009]; amphibians [Roberts and Byme 2011]), including human societies (Scelza 2011). When a few successful males monopolize extrapair paternity in socially monogamous mating systems, the variance in male mating success can be high and potentially lead to strong sexual selection (Gowaty 1985; Westneat et al. 1990; Webster et al. 1995, 2007). However, if most of the variation in male extrapair mating success is generated by ecological or other factors rather than by variation in male traits, then sexual selection will be weak. Accordingly, extrapair paternity can be considered a model for understanding how sexual selection operates and how ecological factors generate variation in mating success.

Most of what we know about the factors influencing extrapair paternity comes from extensive research on how social aspects of bird (Westneat et al. 1990; Griffith et al. 2002; Westneat and Stewart 2003; Wan et al. 2013) and to a lesser extent mammal (Isvaran and Clutton-Brock 2007) populations affect the spatial and temporal opportunities for individuals to encounter and evaluate potential extrapair mates (Bennett and Owens 2002). These aspects include distance between breeding males and females (i.e., breeding distance; Schlicht et al. 2014), breeding density (Westneat et al. 1990; Møller and Birkhead 1993), and reproductive synchrony (Birkhead and Biggins 1987; Westneat et al. 1990; Stutchbury and Morton 1995). High population density and short breeding distances can reduce energetic search costs yet increase the risk of reciprocal cuckoldry (Komdeur 2001; Freeman-Gallant et al. 2005; Canal et al. 2012). Similarly, high synchrony increases the availability of extrapair mates, but its importance is conditional on the female's reproductive stage (Araya-Ajoy et al. 2016) and involves trade-offs among competing male reproductive behaviors, such as mate guarding, parental care, and territory defense (Magrath and Komdeur 2003; While et al. 2011). Social factors have therefore been shown to both promote and constrain extrapair paternity.

The inconsistent effects of these social factors on extrapair paternity (reviewed in Westneat and Sherman 1997; Griffith et al. 2002; Mayer and Pasinelli 2013) may be because studies have rarely been designed to explicitly account for environmental heterogeneity (Westneat and Mays 2005; Rubenstein 2007). Food availability in particular generates spatial and temporal variation in many drivers of reproductive behavior, including population density, timing of breeding, and parental investment decisions. Thus, food availability could influence sexual selection by affecting the distribution of individuals among habitats that differ in quality and the ability of individuals to gain extrapair paternity in those habitats (Rubenstein 2007). Tests of the hypothesis that mating opportunities are jointly influenced by ecological and social factors will therefore advance our understanding of what shapes variation in mating success and the opportunity for sexual selection.

Here, we present a spatially explicit analysis of all possible female-male pairings in a socially monogamous bird to examine the influence of food availability (an ecological factor), breeding density, breeding distance, and the nest stage of a male's social mate (social factors) on extrapair paternity. We evaluate which factors influence the probability that a particular female-male pairing produces extrapair young given the ecological and social conditions they experience. We use genetic parentage data collected from an intensively studied population of black-throated blue warblers, Setophaga caerulescens. Our previous work has shown that extrapair paternity contributes to variation in male reproductive success (Webster et al. 2001) and that food supplementation reduces extrapair paternity (Kaiser et al. 2015), making this population a model system to explore the effects of heterogeneous ecological and social conditions on spatial and temporal opportunities for mating.

\section{Material and Methods \\ Population Monitoring}

We monitored a marked population of black-throated blue warblers over four breeding seasons (2009-2012) at the 3,160-ha Hubbard Brook Experimental Forest in New Hampshire $\left(43^{\circ} 56^{\prime} \mathrm{N}, 71^{\circ} 45^{\prime} \mathrm{W}\right)$. The study area encompassed three study plots at low (250-350 m; 85 ha), middle (450-600 m; $65 \mathrm{ha})$, and high $(750-850 \mathrm{~m} ; 35 \mathrm{ha})$ elevations along a 600-m elevation gradient in continuous northern hardwood forest. Black-throated blue warblers were common breeders in the understory throughout our study area, and their density increased with elevation (Cline et al. 2013). Abundance and biomass of lepidoteran larvae, the warblers' primary food source (Robinson and Holmes 1982; Holmes et al. 1986), were correlated with understory leaf density and also increased from low to high elevations (Rodenhouse et al. 2003).

The breeding ecology of black-throated blue warblers has been studied extensively at Hubbard Brook (Holmes 2007, 2011). Males and females arrive to breed in early May (Holmes et al. 2005). Males defend 1-4-ha territories, with the smallest territories in areas where the shrub layer is dense (Holmes et al. 2005). Hobblebush (Viburnum alnifolium), the preferred nest substrate for the warblers, along with striped maple (Acer pensylvanicum) and American beech (Fagus grandifolia) saplings, were the dominant shrub-layer plants (Schwarz et al. 2003; Holmes et al. 2005). 
All detected males hold territories (Sillett et al. 2004). Females lay one egg per day (mean clutch size $=4$ eggs, range $=2-5$ eggs) and begin incubation when the penultimate egg is laid (Holmes et al. 2005). Females will attempt second broods depending on resource conditions (Nagy and Holmes 2005; Kaiser et al. 2015). Males guard their mates during nest building and egg laying and provision their nestlings (10 days) but attend their nests infrequently while their social mate is incubating (12 days) and rarely feed females at the nest (Holmes et al. 2005).

In each breeding season (May-August), we captured, color banded, and collected blood samples from adults and found and monitored nesting attempts. We caught adults in mist nets and marked each adult with a unique combination of colored leg bands and a US Geological Survey (USGS) aluminum band. Young were marked 2 or 3 days before fledging with a USGS band. At capture, we used plumage characters to classify adults as yearlings or older breeders (Holmes et al. 2005). We collected a small blood sample from the brachial vein of adults $(<70 \mu \mathrm{L})$ and nestlings $(<30 \mu \mathrm{L})$ for parentage assignment. We preserved blood in lysis buffer at $4^{\circ} \mathrm{C}$ until genetic analyses were conducted. We found nests by following females during nest building, following adults carrying food, and searching vegetation. The location of each nest was recorded using a handheld GPS unit. We monitored nests every other day throughout all nest stages and daily near hatch and fledge dates.

\section{Genetic Parentage Analyses}

We genotyped 900 nestlings from 271 broods and nearly all adults ( $>95 \%)$ in the study area at six highly polymorphic microsatellite loci. We extracted DNA from blood samples using DNeasy blood and tissue kits (Qiagen, Valencia, CA). We genotyped adults and nestlings using loci developed from Setophaga caerulescens (Webster et al. 2001), Setophaga petechia (Dawson et al. 1997), Vermivora chrysoptera (Stenzler et al. 2004), and Catharus ustulatus (Gibbs et al. 1999). We amplified $1 \mu \mathrm{L}$ of genomic DNA from each individual at each locus in a $10-\mu \mathrm{L}$ polymerase chain reaction (PCR). The PCR consisted of $6.1 \mu \mathrm{L} \mathrm{dH}_{2} \mathrm{O}, 1 \mu \mathrm{L} \times 10$ PCR buffer (Applied Biosystems, Carlsbad, CA), $1.2 \mu \mathrm{L} 25 \mathrm{mM} \mathrm{MgCl}_{2}$, $0.2 \mu \mathrm{L} 10 \mathrm{mM}$ deoxyribonucleotide triphosphates, $0.2 \mu \mathrm{L}$ $10 \mu \mathrm{M}$ forward (fluorescently labeled) and pigtail reverse primers, and $0.1 \mu \mathrm{L} 2.5 \mathrm{U} \mu \mathrm{L}^{-1}$ Taq DNA polymerase (Invitrogen, Grand Island, NY). PCRs were run on a thermal cycler for 30 cycles, beginning with denaturation at $94^{\circ} \mathrm{C}$ for $1 \mathrm{~min}$, primer-specific annealing temperature $\left(58^{\circ} \mathrm{C}\right)$ for $1 \mathrm{~min}$, and extension at $72^{\circ} \mathrm{C}$ for $45 \mathrm{~s}$ followed by $72^{\circ} \mathrm{C}$ for $5 \mathrm{~min}$. We analyzed the PCR products with a size reference (GeneScan 500 bp LIZ, Applied Biosystems) on an automated 3730 DNA analyzer (Applied Biosystems), and we scored alleles using Genemapper v.3.7 (Applied Biosystems).
We ran paternity analyses using a maximum-likelihood approach for each study plot and breeding season separately; males sampled within and adjacent to the plot boundaries were included as candidate sires. We used CERVUS v.3.0 (Kalinowski et al. 2007) to assign paternity, with the assumption that the social mother was the genetic mother (Webster et al. 2001), which we later verified by examining the frequency of mother-offspring mismatches. We evaluated CERVUS assignments of genetic fathers using trio log-likelihood scores (logarithmic odds; LOD) that are calculated to statistically distinguish among nonexcluded candidate males and consider the genotypes of the known mother and potential mistyping errors. We repeated PCRs at mismatched loci if the social father had a high likelihood score to determine whether mismatches were likely due to genotyping error and whether individuals appeared to be homozygous at a locus because of allelic dropout or a null allele. We accepted CERVUS assignments if the candidate male mismatched offspring at $\leq 1$ locus. However, when two males had similar LOD scores and both had only one mismatch, we also evaluated the CERVUS assignment by considering whether the candidate male was the social father or sired offspring in the same nest. If the social father was excluded as a possible sire by CERVUS (because of a negative LOD score) or mismatched offspring at $\geq 2$ loci, we considered the nestling to have been sired by an extrapair male. The six polymorphic loci yielded a high combined probability of paternal exclusion of 0.999. For our analyses, we excluded broods associated with a separate food-supplementation experiment conducted during the years of this study ( $n=152$ nestlings).

\section{Ecological and Social Factors}

We constructed spatial networks of breeding individuals, including all possible female-male pairings. We mapped the boundaries of male territories throughout the breeding season relative to the $50 \times 50-\mathrm{m}$ grid that delimited each plot. Males were observed every 3 days for 15-20-min periods, and their movements, singing locations, and agonistic interactions with neighboring males were recorded (Holmes et al. 1992; Sillett et al. 2004). At the end of each breeding season, we combined monthly territory maps into one consensus map for the breeding season. Territory boundaries were georeferenced to the plot grid points, territory polygons were digitized, and the geographic coordinates of the centroids and size $\left(\mathrm{m}^{2}\right)$ of each territory (hereafter, territory size) were extracted using ArcGIS 10 (ESRI 2011). We considered the nest site to approximate the location of a female and the centroid of a territory to represent the location of a male. We calculated all pairwise Euclidean distances between each focal female (nest site) and candidate male (territory centroids, excluding female-social mate pairings) by plot and year (hereafter, breeding distance). 
We calculated an index of breeding density for each male as the number of territory centroids of all males that fell within a circular buffer centered at each male's territory centroid. To determine the size of the buffer, we first estimated the mean territory size ( $1.55 \mathrm{ha}$ ) and then calculated the radius of a circle with an area equal to the mean territory size $(\sim 70 \mathrm{~m})$. We estimated breeding density within $70,140,210$, and $280 \mathrm{~m}$. If no male other than the focal male fell within the buffer, we set the breeding density index to 0 ; this occurred for only one male (see "Statistical Analyses" for details on selecting the breeding density index used in our final model).

We examined reproductive synchrony based on the overlap in fertile periods between a focal female's nest and that of a candidate male's social mate to determine which nest stages a male sired extrapair young. The risk of cuckoldry for species with high rates of extrapair paternity begins several days prior to egg laying and extends over most of the egg-laying stage (Birkhead and Møller 1992). Accordingly, we followed Chuang-Dobbs et al. (2001) and defined a female's fertile period to include the 3 days prior to clutch initiation through the day the penultimate egg was laid. We restricted the analysis to the subset of males for which full reproductive data were available for known social mates. We calculated the difference in the clutch completion dates ( $\triangle \mathrm{CCD}$ ) between the extrapair female's nest and that of the male's social mate. When a male's social mate had more than one nest in a season, we used the CCD from the nest that was active nearest in time to the extrapair female's nest. Positive values indicate males that fertilized eggs with extrapair females after the completion of his social mate's clutch; negative values indicate extrapair fertilizations that occurred prior to the completion of his social mate's clutch. We then created the variable nest stage by determining which nest stages a male sired extrapair young through relating the $\Delta \mathrm{CCD}$ to the onset and length of each nest stage of his social mate (i.e., fertile, incubation, nestling, postfledging). Our approach assumes that female black-throated blue warblers do not consistently store viable sperm for more than a few days after a copulation (Johnsen et al. 2012) and that copulations resulting in extrapair fertilizations occur during a female's fertile period (Birkhead and Møller 1992).

We estimated an index of food availability during a focal female's fertile stage for each candidate male's territory from the estimated density of caterpillars on suitable substrates (i.e., the density of leaves on the male's territory) and territory size (Robinson and Holmes 1982; Holmes et al. 1986). We counted and measured caterpillars ( $\mathrm{mm}$ ) on hobblebush and striped maple leaves in the understory along plot-wide transect surveys conducted during six biweekly survey periods (May 15-August 15). Caterpillar measures were converted to wet biomass (mg) using length-mass regressions. We estimated hobblebush and striped maple leaf abundance indexes for each territory from interpolated surfaces of leaf abundance derived from understory leaf sampling (0-3-m height). Field procedures are described in detail elsewhere (Holmes et al. 1979; Holmes and Schultz 1988; Sillett et al. 2004). We summed over all pixels within territory boundaries using ArcGIS 10 (ESRI 2011) and the Geospatial Modelling Environment (Beyer 2012) following Kaiser et al. (2015). We totaled transect estimates of caterpillar biomass (mg leps), averaged per-transect estimates, and divided by 1,000 sampled leaves to obtain the mean caterpillar biomass per leaf for each food substrate in each survey period. For each female-male pairing, we calculated a caterpillar density index as

$$
\begin{gathered}
\left(\text { hobblebush abundance } \times \frac{\mathrm{mg} \text { leps }}{\text { hobblebush leaf }}\right) \\
+\left(\text { striped maple abundance } \times \frac{\mathrm{mg} \mathrm{leps}}{\text { striped maple leaf }}\right),
\end{gathered}
$$

where mg leps per hobblebush leaf and $\mathrm{mg}$ leps per striped maple leaf were equal to the mean from the survey period coinciding with the fertile stage of each focal female.

\section{Statistical Analyses}

Model 1: Testing the Effects of Ecological and Social Factors on $\mathrm{P}_{e p y}$. We constructed a generalized linear mixed model (GLMM) with a binomial distribution and logit link function to test the effects of ecological and social factors and their interactions on the probability of a male siring extrapair young with a female (hereafter, $P_{\text {epy }}$. The response variable was equal to 1 if a male sired extrapair young with a female and 0 otherwise. Analyses were conducted in R, version 3.1.3 (R Development Core Team 2015), with lme4 (Bates et al. 2015), car (Fox and Weisberg 2011), and MuMIn (Barton 2016). ${ }^{1}$ Data underlying models 1 and 2 are deposited in the Dryad Digital Repository: http://dx.doi.org /10.5061/dryad.ck0ts (Kaiser et al. 2016). Our aim was to examine the influence on $P_{\text {epy }}$ of caterpillar abundance within a male's territory (an ecological factor), breeding density, breeding distance, the nest stage a male sired extrapair young relative to his social mate (determined from our index of reproductive synchrony, $\triangle \mathrm{CCD}$; social factors), and the pairwise interactions between caterpillar abundance and the other factors. We considered interactions only hypothesized a priori to affect the movement of individuals or their energetic costs for extrapair paternity. Specifically, we tested for interactions between (1) caterpillar abundance and breed-

1. Code that appears in the American Naturalist is provided as a convenience to the readers. It has not necessarily been tested as part of the peer review. 
ing density to examine whether a combination of high food availability and high breeding density (which may increase female-male encounters) affected $P_{\text {epy }}$ beyond their individual effects (i.e., positive interaction), (2) caterpillar abundance and breeding distance to examine whether males from food-abundant territories travel larger distances to pursue extrapair paternity relative to individuals from food-poor territories because of reduced energetic search costs for mates (i.e., positive interaction), and (3) caterpillar abundance and nest stage to examine whether the effect of food availability differed by nest stage because of differences in energetic trade-offs among nest stages. We included caterpillar abundance in this analysis, which is caterpillar density multiplied by territory size. This is a more parsimonious index of food availability than including the separate covariates for caterpillar density and territory size, in which case the previously described interactions would become three-way interactions with numerous possible two-way interactions, making biological interpretation difficult.

We standardized all model covariates and specified random effects as appropriate. Continuous covariates were standardized to have sample mean equal to 0 and sample variance equal to 1 to improve model convergence. Using this parameterization, the main effect of a covariate that interacts with other covariates is interpreted as the size of its effect on the standard deviation scale at the average value of the terms with which it interacts. We coded factor levels of nest stage using sum-to-zero constraints. Thus, the sum of all nest stage coefficients is equal to 0 . This allows the main effect of the covariate that interacts with nest stage (e.g., caterpillar abundance) to be interpreted as the overall effect across nest stages rather than treating one of the factor levels of nest stage as a reference. Male, female, and nest identities were included as random effects in each model. Male identity accounted for repeated observations of each candidate male in the female-male pairings; female and nest identity accounted for repeated observations of the female in the female-male pairings. We did not include plot as a random effect in the final model because it had only three levels ( $n=3$ study plots; Box 4; Bolker et al. 2009) and because plots were associated with differences in other covariates that were included in the model (caterpillar abundance and breeding density indexes tended to be higher for the high-elevation plot). Moreover, when plot was included in the final model as a random intercept, its estimated variance was equal to 0 .

Prior to hypothesis testing, we had three model-selection steps: (1) choose the best-supported breeding density index; (2) explore log, linear, and quadratic functions of breeding distance; and (3) select nuisance covariates. We used the Akaike information criterion corrected for finite sample sizes (AICc) to guide our choice of breeding density measured at four spatial scales (one to four times the radius of a circle corresponding to the average territory size). As a base model in this assessment, we included log breeding distance, caterpillar abundance, nest stage, caterpillar abundance : $\log$ breeding distance, caterpillar abundance : nest stage, and the candidate male age class (defined as yearling $=$ 1 , older breeder $=0$ ). These represent the minimum terms to test our hypotheses and one nuisance covariate, which was previously found to influence extrapair paternity in this population (Kaiser et al. 2015). We then compared models with each measure of breeding density and its interaction with caterpillar abundance. We found similar support for breeding density measured within 210 and $280 \mathrm{~m}$ (AICc $=890.43$ and 890.33), and less support for breeding density measured within $70(\mathrm{AICc}=892.68)$ and 140 $($ AICc $=894.32) \mathrm{m}$. We conservatively used breeding density measured within $210 \mathrm{~m}$ to reduce possible edge effects and defined this buffer area as the local neighborhood. Next, we explored whether linear or quadratic effects of breeding distance were better supported than log breeding distance. We compared the model chosen in the first step with the following two models: (1) breeding distance, breeding density within $210 \mathrm{~m}$, caterpillar abundance, nest stage, caterpillar abundance: breeding distance, caterpillar abundance: nest stage, breeding density within $210 \mathrm{~m}$ : caterpillar abundance, and candidate male age and (2) breeding distance, breeding distance ${ }^{2}$, breeding density within $210 \mathrm{~m}$, caterpillar abundance, nest stage, caterpillar abundance: breeding distance, caterpillar abundance : nest stage, breeding density within $210 \mathrm{~m}$ : caterpillar abundance, and candidate male age. The model containing log breeding distance $(\mathrm{AICc}=890.44$; table A2; tables A1, A2 are available online) was better supported than models with breeding distance (AICc $=917.98)$ or breeding distance and breeding distance $^{2}(\mathrm{AICc}=895.95)$. These steps determined our base model, which also included a covariate previously found to affect extrapair paternity (candidate male age), the covariates of interest (caterpillar abundance, breeding density within $210 \mathrm{~m}, \log$ breeding distance), and the interactions of interest (caterpillar abundance : breeding density within $210 \mathrm{~m}$, caterpillar abundance: $\log$ breeding distance, and caterpillar abundance: nest stage).

We used AICc to guide whether to include several additional covariates that we considered nuisance variables because they did not directly pertain to our research aims: social male age (age class of the focal female's social mate), clutch completion date (CCD) of the first nest of the candidate male to account for potential seasonality effects, and the following interactions: caterpillar abundance: candidate male age and log breeding distance: candidate male age. The candidate models are listed in table A1. No single model was better supported; therefore, we conducted model averaging on all models in table A1. However, the model-averaged estimates and standard errors were very similar to the single-model pa- 
rameter estimates and errors (described below). We elected to use a single model in subsequent analyses to conduct classical hypothesis testing using Wald tests ( $z$ scores) of individual coefficients and likelihood ratio tests (LRTs) to test the significance of covariates and their interactions. We used LRTs to evaluate the overall influence of each hypothesized fixed effect and its interactions by comparing the selected model to reduced models excluding the main effect and its interactions. The selected model containing the base model and log breeding distance: candidate male age was nearly as well supported as the top-ranked model, which included social male age $(\Delta \mathrm{AICc}=0.28)$. However, the top-ranked model with social male age, based on AICc, had a higher Bayesian information criterion $(\mathrm{BIC}=1,021.31)$ than the selected model $(\mathrm{BIC}=1,008.56)$, and the LRT between these two models was not significant $\left(\chi_{2}^{2}=4.3, P=.12\right)$. Given the substantially lower BIC and the equivocal evidence from AICc, we used the model without social male age.

We diagnosed potential issues with multicollinearity in the selected model by examining the variance inflation factors and generalized variance inflation factors for categorical variables (Fox and Monette 1992), and we also examined the approximate correlation matrixes of the parameter estimates. Note that centering standardized covariates reduces potential issues with the fixed effects correlation matrix (Zuur et al. 2009). In the final model, the maximum VIF was 4.1 (caterpillar abundance), which is below common multicollinearity thresholds (e.g., 10 in Kutner et al.2004) but above more conservative thresholds (e.g., 3 in Zuur et al. 2009). The maximum correlation between main effects was -0.49 (candidate male age and log breeding distance), and the maximum correlation including interaction effects was -0.69 (candidate male age and log breeding distance: candidate male age).

Model 2: Testing the Effect of Food Availability on Breeding Distance. Next, we examined whether food availability influenced the distance between the territory of a male and the nests in which he sired extrapair young using a linear mixed model (LMM) with log breeding distance as the response variable. We restricted this analysis to include female-male pairings that produced extrapair young. We included candidate male age, caterpillar density (measured during the extrapair female's fertile stage), territory size, and caterpillar density : territory size as fixed effects and included nest identity as a random effect. As in the previous model, all continuous variables were standardized. Note that for this analysis we decomposed caterpillar abundance into caterpillar density and territory size. $P$ values of approximate $t$ statistics were estimated with Satterthwaite's approximation using lmerTest (Kuznetsova et al. 2013). The maximum VIF was 1.86 (caterpillar density), the maximum correlation between the main fixed effects was 0.29 (territory size and caterpillar density), and the VIF among all fixed effects was
0.64 (correlation between caterpillar density : territory size and caterpillar density).

\section{Results \\ Summary Results}

We assigned paternity to 821 of 900 (91.2\%) genotyped nestlings. We assigned social fathers to 505 offspring (56.1\%) and 316 offspring to extrapair males (35.1\%); 79 offspring $(8.8 \%)$ that mismatched their social fathers could not be assigned to any male and were considered extrapair young. The paternity analyses revealed that 395 offspring (43.4\%) were extrapair young and 152 nests (55.6\%) contained at least one nestling sired by an extrapair male. The number of extrapair young sired by males ranged from zero to eight (mean $=0.94$, variance $=2.07$ ). The proportion of males that sired at least one extrapair young was 0.45 . Restricting the analysis to candidate males with complete nest data and no missing data, 107 candidate female-male pairings resulted in extrapair paternity out of 5,030 possible female-male pairs, comprised of 200 females, 187 males, and 253 nests. In this data set, the proportion of males that sired at least one extrapair young was 0.39 . Of the 107 female-male pairings resulting in extrapair young, $15,18,55,9$, and 10 occurred during the prefertile, fertile, incubation, nestling, and fledgling stages of the candidate male's social mate, respectively, and these observed counts were significantly different from expected given the nest stage frequencies among the 5,030 possible female-male pairs $\left(\chi_{4}^{2}=14.58, P=.006\right.$; does not account for repeated observations on males, females, or nests).

Model 1: Testing the Effects of Ecological and Social Factors on $\mathrm{P}_{\text {epy. }}$ Multiple ecological and social factors and their interactions were related to the probability of a male siring extrapair young with a female $\left(P_{\text {epy }}\right)$. A female's proximity to the male's territory was positively related to $P_{\text {epy }}$ (fig. 1; log breeding distance; table 1), and the overall effect of log breeding distance, including its pairwise interactions with candidate male age and caterpillar abundance, significantly improved model fit (likelihood ratio test [LRT]: $\chi_{3}^{2}=149.75$, $P<.001) . P_{\text {epy }}$ decreased with increasing breeding density (breeding density within $210 \mathrm{~m}$; table 1), and the main effect was significant $(P=.027)$, which represents an effect size significantly different from 0 at the average caterpillar abundance. The interaction between caterpillar abundance and breeding density was not significant $(P=.97$; table 1$)$, and the overall effect of breeding density, including its interaction with caterpillar abundance, did not significantly improve model fit (LRT: $\left.\chi_{2}{ }_{2}=4.94, P=.085\right) . P_{\text {epy }}$ was initially lower and also decreased less rapidly with breeding distance for yearling males compared to older males (fig. 1; 


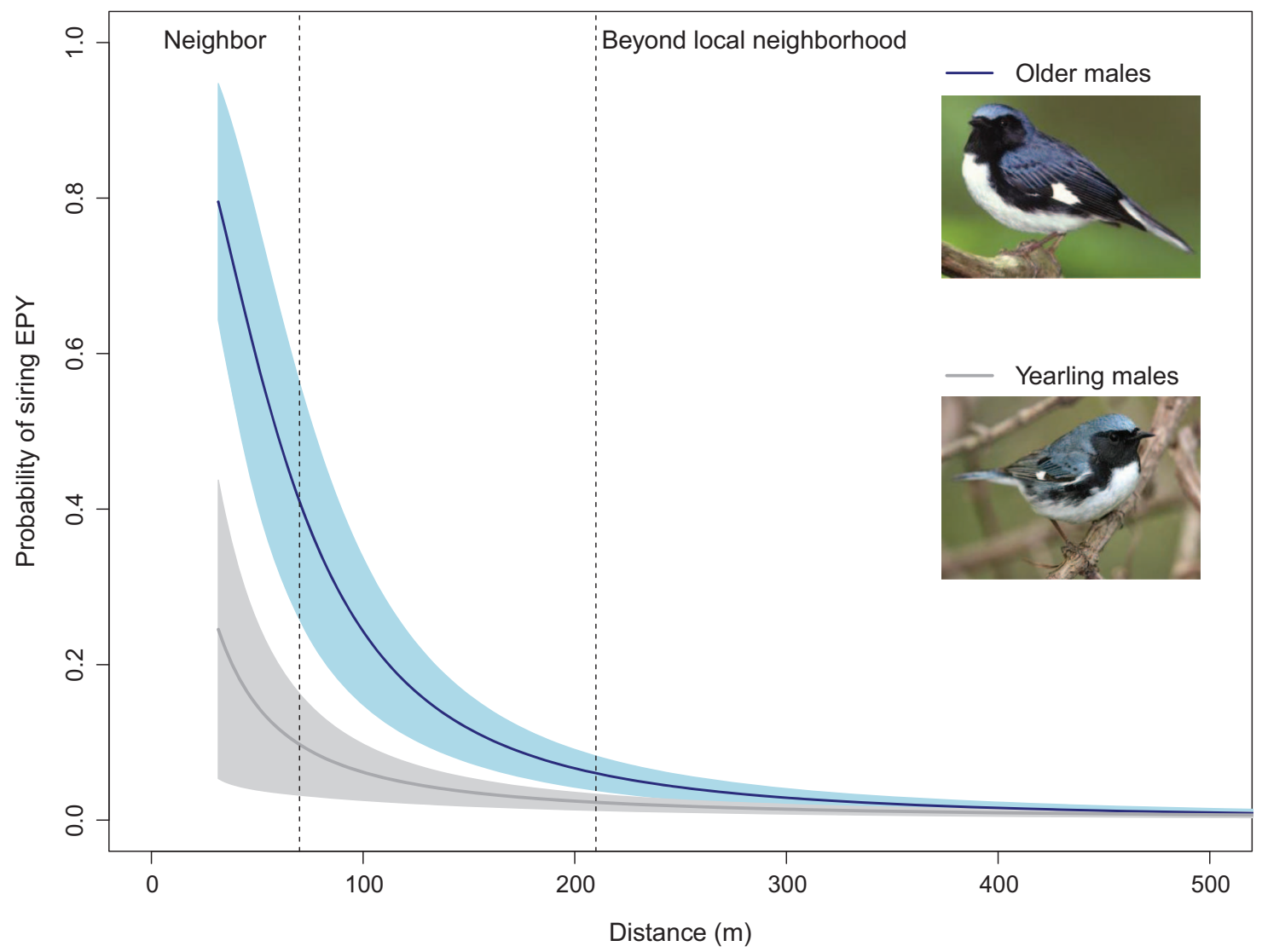

Figure 1: Effect of breeding distance between females (nest sites) and each candidate male (territory centroids, excluding female-social mate pairings) on the probability of a male siring extrapair young (EPY) with $95 \%$ confidence bands by male age class (gray line $=$ yearlings and blue line $=$ older males) in the black-throated blue warbler. We converted the distance effect sizes from the logit scale to probabilities as a function of distance in meters for yearling and older males. We used the delta method to calculate $95 \%$ confidence intervals for the effect of male age class and breeding distance on the probability of siring extrapair young while setting all other covariates equal to their mean and setting the random effects to zero. The local neighborhood was defined as an area equal to three times the mean male territory size (1.55 ha; i.e., a circular buffer with radius $=210 \mathrm{~m}$ ) and a neighbor was within $70 \mathrm{~m}$. Photo credits: top photo, Arthur Morris; bottom photo, Christopher Taylor.

$\log$ breeding distance : candidate male age; $P<.01$; table 1 ). The difference in $P_{\text {epy }}$ was not significant between age classes at the average log breeding distance (candidate male age; $P=.27$; table 1 ; note that mean log breeding distance $=$ 6.21 or $495 \mathrm{~m}$ ). However, the overall effect of male age, including its interaction with $\log$ breeding distance on $P_{\text {epy }}$, significantly improved model fit (LRT: $\chi_{2}{ }_{2}=21.12, P<.001$ ). Males were more likely to sire extrapair young during their social mate's incubation stage compared to the mean across nest stages (fig. 2; nest stage [incubation]; table 1), and the overall effect of nest stage on $P_{\text {epy }}$ including its interaction with caterpillar abundance, significantly improved model fit (LRT: $\chi_{8}^{2}=18.44, P=.018$ ).

Food availability was broadly associated with spatial and temporal patterns of extrapair paternity. $P_{\text {epy }}$ decreased with increasing caterpillar abundance within a male's territory during the focal female's fertile stage (caterpillar abundance; table 1). The main effect of caterpillar abundance on $P_{\text {epy }}$ was significant $(P=.006)$, which indicates that its effect was significantly different from 0 at the average value of the variables with which it interacted. The overall effect of caterpillar abundance, including its interactions with log breeding distance, breeding density, and nest stage (involving a loss of six degrees of freedom), did not significantly improve model fit (LRT: $\left.\chi^{2}{ }_{7}=13.32, P=.065\right)$. The decrease in $P_{\text {epy }}$ with breeding distance was more rapid for males on foodabundant territories than for those on food-poor territories (caterpillar abundance: log breeding distance; table 1). Males on food-abundant territories were more likely to sire extrapair young during their social mate's incubation stage than those on food-poor territories (caterpillar abundance: nest stage [incubation]; table 1). 
Table 1: Model testing hypothesized effects of ecological and social factors and their interactions on the probability of a male siring extrapair young in the black-throated blue warbler

\begin{tabular}{|c|c|c|c|}
\hline \multirow[b]{2}{*}{ Effect and model term ( $n=5,030$ female/candidate male-year pairings) } & \multicolumn{3}{|c|}{ Probability of EPY } \\
\hline & $\beta \pm \mathrm{SE}$ & $z$ & $P$ \\
\hline \multicolumn{4}{|l|}{ Fixed effects: } \\
\hline Intercept & $-4.63 \pm .27$ & -17.10 & $<.001$ \\
\hline Candidate male age (yearling) & $-.31 \pm .28$ & -1.10 & .27 \\
\hline Log breeding distance ${ }^{\mathrm{a}}$ & $-1.28 \pm .13$ & -9.81 & $<.001$ \\
\hline Breeding density ${ }^{\mathrm{b}}$ & $-.26 \pm .12$ & -2.21 & .027 \\
\hline Nest stage $(\text { fertile })^{c}$ & $-.54 \pm .30$ & -1.82 & .069 \\
\hline Nest stage (incubation) & $.58 \pm .18$ & 3.26 & .001 \\
\hline Nest stage (nestling) & $.00 \pm .31$ & -.01 & .99 \\
\hline Nest stage (postfledging) & $.14 \pm .30$ & .47 & .64 \\
\hline Caterpillar abundance ${ }^{\mathrm{d}}$ & $-.64 \pm .23$ & -2.77 & .006 \\
\hline Log breeding distance : candidate male age (yearling) & $.47 \pm .17$ & 2.72 & .006 \\
\hline Caterpillar abundance : log breeding distance & $-.22 \pm .11$ & -2.04 & .041 \\
\hline Caterpillar abundance : breeding density & $.00 \pm .12$ & .04 & .97 \\
\hline Caterpillar abundance : nest stage (fertile) & $-.54 \pm .43$ & -1.26 & .21 \\
\hline Caterpillar abundance : nest stage (incubation) & $.42 \pm .20$ & 2.09 & .037 \\
\hline Caterpillar abundance : nest stage (nestling) & $.03 \pm .38$ & .07 & .95 \\
\hline Caterpillar abundance : nest stage (postfledging) & $.18 \pm .31$ & .59 & .56 \\
\hline Random effects & Variance & $n$ & \\
\hline Nest identity & $<.01$ & 253 & \\
\hline Female identity & .30 & 200 & \\
\hline Male identity & .00 & 187 & \\
\hline
\end{tabular}

Note: Significant fixed effects are shown in boldface. Log breeding distance, breeding density, and caterpillar abundance were standardized to have sample mean $=0$ and sample variance $=1$. EPY $=$ extrapair young.

${ }^{a}$ Breeding distance $=$ Euclidean distance between each female (nest site) and each candidate male (territory centroids, excluding female-social mate pairings).

${ }^{\mathrm{b}}$ Breeding density $=$ number of candidate males (territory centroids) that fell within a circular buffer centered on the focal male's territory with a radius equal to three times the radius corresponding to the mean male territory size ( 1.55 ha; i.e., radius $=210 \mathrm{~m}$ ).

${ }^{\mathrm{c}}$ Nest stage $=$ the nest stage a male sired extrapair young relative to their social mate was determined by relating the difference in clutch completion dates between a male's social mate and his extrapair female ( $\triangle \mathrm{CCD}$; index of reproductive synchrony) to the onset and length of each nest stage of his social mate (i.e., fertile, incubation, nestling, postfledging). We coded factor levels using sum-to-zero constraints.

${ }^{\mathrm{d}}$ Caterpillar abundance $=$ caterpillar density estimated on a male's territory from the survey period coinciding with the fertile stage of each female multiplied by male territory size.

Model 2: Testing the Effect of Food Availability on Breeding Distance. Last, males on territories with higher caterpillar density sired extrapair young closer to their own territories than did males on food-poor territories (LMM: $\beta \pm$ SE: $\left.-0.32 \pm 0.13, t_{98}=-2.55, P=.013\right)$, but log breeding distance was not associated with the size of a candidate male's territory (LMM: $\beta \pm$ SE: $0.13 \pm 0.10, t_{98.4}=1.29$, $P=.20)$ or the interaction between territory size and caterpillar density (LMM: $\beta \pm$ SE: $-0.24 \pm 0.13, t_{100.3}=-1.83$, $P=.071)$. Yearling males sired extrapair young at greater distances from their own territories than older males (LMM: $\beta \pm$ SE: $\left.0.43 \pm 0.20, t_{106}=2.22, P=.029\right)$.

\section{Discussion}

The inability of sexual traits to explain variation in male mating success in numerous empirical studies (Westneat
2006; Akçay and Roughgarden 2007; Soulsbury 2010; Hsu et al. 2015) implies that the strength of sexual selection in natural populations is moderated by other factors. We show here, using a spatially explicit, multifactor model of extrapair paternity, that an individual's mating success is affected by both ecological and social factors. Most extrapair mating opportunities were spatially limited to a male's local neighborhood and temporally limited to the incubation stage of a male's social mate. These constraints were more pronounced for males on food-abundant territories. Food availability generated unequal spatial and temporal opportunities for extrapair paternity and influenced the distribution of mating success among individuals. Our results therefore support the hypothesis that mating opportunities are jointly influenced by ecological and social factors and suggest that the strength of sexual selection may be moderated by these factors and that sexual selection dy- 


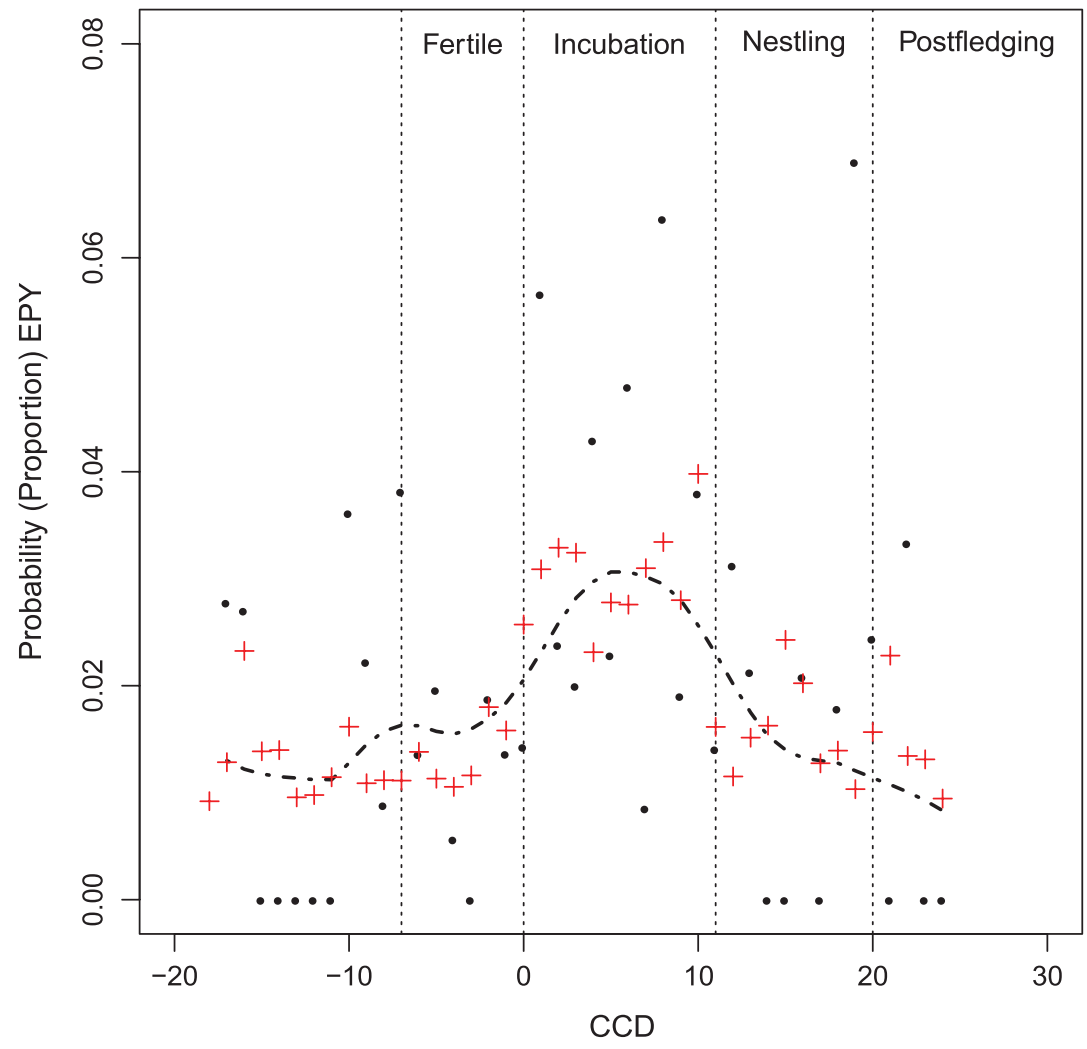

Figure 2: Proportion of female-male pairings that produced extrapair young for each $\triangle \mathrm{CCD}$ (difference in clutch completion dates between a candidate male's social mate and the potential extrapair female; black dots) and the predicted probabilities from the model in table 1 averaged across female-male pairings by $\triangle \mathrm{CCD}$ (red plus symbols) in the black-throated blue warbler. Positive values indicate that the male's social mate had completed her clutch before the extrapair female; negative values indicate the opposite. We determined which nest stages a male sired extrapair young relative to their social mate by relating the $\triangle \mathrm{CCD}$ to the onset and length of each nest stage of his social mate (i.e., fertile, incubation, nestling, postfledging). The curve was fitted to the observed proportions with a loess smoother using locally weighted polynomial regression and is included here to aid visualization of the nonlinear effect of $\triangle \mathrm{CCD}$. Note that our model captures the nonlinear effect using an indicator variable for each nest stage (i.e., a piecewise constant function, which facilitates the testing of our biological hypotheses). The vertical dashed lines indicate the onset and length of each nest stage of the social mate.

namics may vary with heterogeneous ecological and social conditions.

Food availability has long been shown to be associated with reproductive success (Lack 1954; Martin 1987; Sinclair 1989; Newton 1998), but existing hypotheses propose conflicting explanations about its influence on spatial and temporal opportunities for extrapair paternity (Gowaty 1996; Hoi-Leitner et al. 1999; Rubenstein 2007; Kaiser et al. 2015). The commonly invoked energetic cost hypothesis asserts that greater food abundance allows males more time and energy to pursue extrapair paternity (Magrath and Komdeur 2003; Westneat and Stewart 2003). We found that overall, males on territories with abundant food during a potential extrapair female's fertile stage had a lower probability of siring extrapair young (main effect; table 1), which is contrary to the energetic cost hypothesis. However, food availability on a candidate male's territory while his social mate was in- cubating increased his probability of siring extrapair young, which supports the energetic cost hypothesis. Males generally sired extrapair young in nearby females' nests and while their social mate was incubating. Males on food-poor territories, in contrast, had a higher probability of siring extrapair young beyond their local neighborhood. These findings support the foraging-encounter hypothesis, which proposes that individuals on food-poor territories have more opportunities to encounter extrapair mates because they move larger distances to forage (Gray 1997). Although we did not collect movement data, some bird and mammal studies have shown that individuals on food-poor territories forage farther than individuals on food-abundant territories (e.g., Rubenstein 2007; Cameron et al. 2011), and pairs provided supplemental food generally spend more time near their nests and in territorial defense (Westneat 1994; Václav et al. 2003; O’Brien and Dawson 2011; Kaiser et al. 2014). Our results 
are consistent with studies that have examined spatial and temporal patterns in extrapair paternity at the individual level (Canal et al. 2012; Taff et al. 2013; García-Navas et al. 2014; Schlicht et al. 2014; but see Araya-Ajoy et al. 2016). However, we also demonstrate that variation in food availability affects spatial and temporal patterns in extrapair mating success. This relationship between extrapair paternity and habitat quality has implications for sexual selection if extrapair paternity has a strong effect on overall variance in male lifetime reproductive success (Webster et al. 1995, 2007).

The extent to which extrapair paternity increases the opportunity for sexual selection is controversial (Webster et al. 2007). Extrapair paternity could have small effects on the opportunity for sexual selection if reciprocal cuckoldry or behavioral trade-offs cancel extrapair paternity gains (Hasselquist and Sherman 2001; Jones et al. 2001; Freeman-Gallant et al. 2005) or if most males, rather than only a small subset of highly successful males, are able to sire at least some extrapair young (Pedersen et al. 2006; Sousa and Westneat 2013). Our previous work on black-throated blue warblers shows that extrapair paternity can increase the variance in mating success, although the magnitude of this effect is weak and varies between years (Webster et al. 2001). Most variance was generated by differences in within-pair paternity because black-throated blue warblers can raise two broods in a season but only one-third of the population is successful at double brooding (Webster et al. 2001; Townsend et al. 2013). Moreover, a positive covariance between extrapair and within-pair paternity indicated that males that gain extrapair paternity were also less likely to be cuckolded (Webster et al. 2001), further increasing the variance in mating success and hence the opportunity for sexual selection (Møller and Birkhead 1994; Webster et al. 1995; Møller and Ninni 1998; Reid et al. 2014). Despite the opportunity for sexual selection and striking sexual dichromatism (Holmes et al. 2005; Cline et al. 2016), male sexual traits do not appear to be associated with extrapair or within-pair mating success in our study population (Webster et al. 2001; M. S. Webster, unpublished data). This finding parallels that of other studies that have failed to find associations between sexual signals and extrapair mating success (e.g., Soulsbury 2010; Hsu et al. 2015).

One possibility for weak evidence that extrapair paternity leads to sexual selection on male traits in numerous studies is that much of the variance in male mating success is generated by ecological and social factors rather than by differences in male traits. In this study, we demonstrate how ecological and social factors can limit mating opportunities and affect variation in male mating success. First, we show that a male's probability of siring extrapair young decreased with increasing distance to females. Extrapair paternity often occurs between close neighbors because these individuals have the highest probability of interacting with each other (Yezerinac et al. 1995; Chuang et al. 1999; Pedersen et al. 2006; Schlicht et al. 2014). When females limit their mate choice to nearest neighbors, the pool of potential mates is constrained, which reduces variance in male mating success (Webster et al. 1995, 2001; Whittingham and Dunn 2005; Taff et al. 2013). Second, we show that a male's probability of siring extrapair young decreased with increasing breeding density, although these effects were relatively weak compared to other studies (reviewed in Westneat and Sherman 1997) and the other factors examined. High local breeding density can reduce extrapair mating success within a local neighborhood because of increased male-male competition and risk of reciprocal cuckoldry (Schlicht et al. 2014). Third, we show that a male's probability of siring extrapair young increased during their social mate's incubation stage. This finding supports the trade-off avoidance hypothesis, which proposes that males avoid pursuing extrapair paternity when they face trade-offs with mate guarding (fertile stage) and parental care (nestling and postfledging stages; Westneat et al. 1990). Most studies examining the temporal constraints of breeding synchrony have focused on how the degree of overlap of females' fertile periods affects male mating opportunities (i.e., trade-offs with mate guarding) at the population or local scales (e.g., Kempenaers 1993; Chuang et al. 1999; but see Araya-Ajoy et al. 2016). We provide the first evidence that the nest stage of a male's social mate limits the temporal opportunity for extrapair paternity. Fourth, we show that a male's probability of siring extrapair young decreased with increasing food availability (caterpillar abundance). Moreover, food availability influenced the spatial and temporal distribution of extrapair paternity among males in food-poor and food-abundant habitat.

Our results also suggest that heterogeneity in ecological conditions may cause the strength of selection to fluctuate over space and time. Evidence is accumulating that sexual selection can vary across environments (Cornwallis and Uller 2010), but studies that examine the mechanisms that underlie spatial and temporal dynamics of sexual selection are rare, especially at fine scales within populations (Clark and Backwell 2015; Janicke et al. 2015). Climate-induced differences in sexual selection across years or populations have been observed in several species (Twiss et al. 2007; Gosden and Svensson 2008; Olsson et al. 2011; Botero and Rubenstein 2012; Robinson et al. 2012). We know less about how climate and other ecological factors could produce fine-scale variation in sexual selection over space and time (Cornwallis and Uller 2010).

The widespread occurrence of extrapair paternity in vertebrates and its remarkable variation (Avise et al. 2002; Griffith et al. 2002; Uller and Olsson 2008; Cohas and Allainé 2009; Roberts and Byme 2011) make it a useful model for understanding how ecological and social factors influence mating success. Extrapair paternity can increase the 
variance in male mating success (Webster et al. 1995, 2007) and for this reason is often an important source of sexual selection in socially monogamous species (Webster et al. 2007). This study demonstrates that both ecological and social factors affect the spatial and temporal opportunities for extrapair paternity. These factors interact to generate variation in male mating success, which could moderate the strength of sexual selection in natural populations. This idea could be tested by conducting experimental manipulations of ecological and social conditions to evaluate their impact on the variance in mating success. By modeling the ecological and social context of extrapair paternity, we were able to determine how different conditions shape individuals' spatial and temporal opportunities for extrapair paternity that likely drive the strength of sexual selection in socially monogamous birds and other species.

\section{Acknowledgments}

We are very grateful to the undergraduates and field technicians that contributed their efforts to this study and to A. Branesky and K. Grabenstein for laboratory assistance. We thank L. Stenzler for technical advice on microsatellite genotyping. We especially thank our collaborators, R. T. Holmes and N. L. Rodenhouse, for their guidance and support. We thank T. Martin, R. Fleischer's lab group, the Editor, Associate Editor, and two anonymous reviewers for valuable comments that greatly improved the manuscript. All research activities were performed under protocols approved by the Animal Care and Use Committees of the authors' institutions. This research was supported by US National Science Foundation (NSF) grants awarded to Cornell University (0640470), the Smithsonian Institution (0640732), and Wellesley College (064082300), by fellowships and grants awarded to S.A.K. from the US Environmental Protection Agency (91723201), Smithsonian Institution, Animal Behavior Society, Sigma Xi, American Ornithologists' Union, Wilson Ornithological Society, and Cornell Lab of Ornithology. B.B.R. was partially supported by an NSF grant awarded to the Statistical and Applied Mathematical Sciences Institute (DMS-1127914). This research is a contribution of the Hubbard Brook Ecosystem Study, part of the Long-Term Ecological Research network supported by the NSF.

\section{Literature Cited}

Akçay, E., and J. Roughgarden. 2007. Extra-pair paternity in birds: review of the genetic benefits. Evolutionary Ecology Research 9:855868.

Andersson, M. 1994. Sexual selection. Princeton University Press, Princeton, NJ.

Andersson, M., and L. W. Simmons. 2006. Sexual selection and mate choice. Trends in Ecology and Evolution 21:296-302.
Araya-Ajoy, Y. G., N. J. Dingemanse, and B. Kempenaers. 2016. Timing of extrapair fertilizations: within-pair fertilization trade-offs or pair synchrony spillovers? Behavioral Ecology 27:377-384.

Arnold, S. J., and M. J. Wade. 1984. On the measurement of natural and sexual selection: theory. Evolution 38:709-719.

Avise, J. C., A. G. Jones, D. Walker, and J. A. DeWoody. 2002. Genetic mating systems and reproductive natural histories of fishes: lessons for ecology and evolution. Annual Review of Genetics 36:19-45.

Bartoń, K. 2016. MuMIn: multi-model inference (R package).

Bates, D., M. Maechler, B. Bolker, and S. Walker. 2015. Fitting linear mixed-effects using lme4. Journal of Statistical Software 67:1-48.

Bennett, P. M., and I. P. F. Owens. 2002. Evolutionary ecology of birds: life histories, mating systems and extinction. Oxford University Press, Oxford.

Beyer, H. L. 2012. Geospatial Modelling Environment. Version 0.7.2.0. http://www.spatialecology.com/gme.

Birkhead, T. R., and J. D. Biggins. 1987. Reproductive synchrony and extra-pair copulation in birds. Ethology 74:320-334.

Birkhead, T. R., and A. P. Møller. 1992. Sperm competition in birds: evolutionary causes and consequences. Academic Press, London.

Bolker, B. M., M. E. Brooks, C. J. Clark, S. W. Geange, J. R. Poulsen, M. H. H. Stevens, and J.-S. S. White. 2009. Generalized linear mixed models: a practical guide for ecology and evolution. Trends in Ecology and Evolution 24:127-135.

Botero, C. A., and D. R. Rubenstein. 2012. Fluctuating environments, sexual selection and the evolution of flexible mate choice in birds. PLoS ONE 7:e32311.

Cameron, C., D. Berteaux, and F. Dufresne. 2011. Spatial variation in food availability predicts extrapair paternity in the arctic fox. Behavioral Ecology 22:1364-1373.

Canal, D., R. Jovani, and J. Potti. 2012. Male decisions or female accessibility? spatiotemporal patterns of extra pair paternity in a songbird. Behavioral Ecology 23:1146-1153.

Chuang, H. C., M. S. Webster, and R. T. Holmes. 1999. Extrapair paternity and local synchrony in the black-throated blue warbler. Auk 116:726-736.

Chuang-Dobbs, H. C., M. S. Webster, and R. T. Holmes. 2001. The effectiveness of mate guarding by male black-throated blue warblers. Animal Behaviour 12:541-546.

Clark, H. L., and P. R. Y. Backwell. 2015. Temporal and spatial variation in female mating preferences in a fiddler crab. Behavioral Ecology and Sociobiology 69:1779-1784.

Cline, M. H., J. L. Hatt, M. J. Conroy, and R. J. Cooper. 2016. Experimental evidence for a phenotypic trait as an age-dependent intrasexual social signal between familiar individuals. Animal Behaviour 111:319-327.

Cline, M. H., A. M. Strong, T. S. Sillett, N. L. Rodenhouse, and R. T. Holmes. 2013. Correlates and consequences of breeding dispersal in a migratory songbird. Auk 130:742-752.

Cohas, A., and D. Allainé. 2009. Social structure influences extrapair paternity in socially monogamous mammals. Biology Letters 5:313-316.

Cornwallis, C. K., and T. Uller. 2010. Towards an evolutionary ecology of sexual traits. Trends in Ecology and Evolution 25:145-152.

Dawson, R. J. G., H. L. Gibbs, K. A. Hobson, and S. M. Yezerinac. 1997. Isolation of microsatellite DNA markers from a passerine bird, Dendroica petechia (the yellow warbler), and their use in population studies. Heredity 79:506-514.

ESRI (Environmental Systems Research Institute). 2011. ArcGIS Desktop: release 10. Environmental Systems Research Institute, Redlands, CA. 
Fox, J., and G. Monette. 1992. Generalized collinearity diagnostics. Journal of the American Statistical Association 87:178-183.

Fox, J., and S. Weisberg. 2011. An R companion to applied regression. 2nd ed. Sage, Thousand Oaks, CA.

Freeman-Gallant, C. R., N. T. Wheelwright, K. E. Meiklejohn, S. L. States, and S. V Sollecito. 2005. Little effect of extrapair paternity on the opportunity for sexual selection in savannah sparrows (Passerculus sandwichensis). Evolution 59:422-430.

García-Navas, V., E. S. Ferrer, J. Bueno-Enciso, R. Barrientos, J. J. Sanz, and J. Ortego. 2014. Extrapair paternity in Mediterranean blue tits: socioecological factors and the opportunity for sexual selection. Behavioral Ecology 25:228-238.

Gibbs, H. L., L. M. Tabak, and K. Hobson. 1999. Characterization of microsatellite DNA loci for a Neotropical migrant songbird, the Swainson's thrush (Catharus ustulatus). Molecular Ecology 8:15511561.

Gosden, T. P., and E. I. Svensson. 2008. Spatial and temporal dynamics in a sexual selection mosaic. Evolution 62:845-856.

Gowaty, P. A. 1985. Multiple parentage and apparent monogamy in birds. Pages 11-21 in P. A. Gowaty and D. W. Mock, eds. Avian monogamy. American Ornithologists Union, Washington, DC.

- 1996. Battles of the sexes and origins of monogamy. Pages 2152 in J. M. Black, ed. Partnership in birds: the study of monogamy. Oxford University Press, Oxford.

Gray, E. M. 1997. Female red-winged blackbirds accrue material benefits from copulating with extra-pair males. Animal Behaviour 53:625-639.

Griffith, S. C., I. P. F. Owens, and K. A. Thuman. 2002. Extra pair paternity in birds: a review of interspecific variation and adaptive function. Molecular Ecology 11:2195-2212.

Hasselquist, D., and P. W. Sherman. 2001. Social mating systems and extrapair fertilizations in passerine birds. Behavioral Ecology 12: 457-466.

Hoi-Leitner, M., H. Hoi, M. Romero-Pujante, and F. Valera. 1999. Female extra-pair behaviour and environmental quality in the serin (Serinus serinus): a test of the "constrained female hypothesis." Proceedings of the Royal Society B 266:1021-1026.

Holmes, R. T. 2007. Understanding population change in migratory songbirds: long-term and experimental studies of Neotropical migrants in breeding and wintering areas. Ibis 149:2-13.

- 2011. Avian population and community processes in forest ecosystems: long-term research in the Hubbard Brook experimental forest. Forest Ecology and Management 262:20-32.

Holmes, R. T., N. L. Rodenhouse, and T. S. Sillett. 2005. Black-throated blue warbler (Setophaga caerulescens), no. 87. In A. Poole, ed. The Birds of North America Online. Cornell Lab of Ornithology, Ithaca, NY. https://birdsna.org/species-account/bna/species/btbwar/.

Holmes, R. T., and J. C. Schultz. 1988. Food availability for forest birds: effects of prey distribution and abundance on bird foraging. Canadian Journal of Zoology 66:720-728.

Holmes, R. T., J. C. Schultz, and P. Nothnagle. 1979. Bird predation on forest insects: an exclosure experiment. Science 206:462-463.

Holmes, R. T., T. W. Sherry, P. P. Marra, and K. E. Petit. 1992. Multiple brooding and productivity of a Neotropical migrant, the blackthroated blue warbler (Dendroica caerulescens), in an unfragmented temperate forest. Auk 109:321-333.

Holmes, R. T., T. W. Sherry, and F. W. Sturges. 1986. Bird community dynamics in a temperate deciduous forest: long-term trends at Hubbard Brook. Ecological Monographs 56:201-220.
Hsu, Y.-H., J. Schroeder, I. Winney, T. Burke, and S. Nakagawa. 2015. Are extra-pair males different from cuckolded males? a case study and a meta-analytic examination. Molecular Ecology 24:1558-1571.

Isvaran, K., and T. Clutton-Brock. 2007. Ecological correlates of extra-group paternity in mammals. Proceedings of the Royal Society B 274:219-224.

Janicke, T., P. David, and E. Chapuis. 2015. Environment-dependent sexual selection: Bateman's parameters under varying levels of food availability. American Naturalist 185:756-768.

Johnsen, A., K. L. Carter, K. Delhey, J. T. Lifjeld, R. J. Robertson, and B. Kempenaers. 2012. Laying-order effects on sperm numbers and on paternity: comparing three passerine birds with different life histories. Behavioral Ecology and Sociobiology 66:181-190.

Jones, A. G. 2009. On the opportunity for sexual selection, the Bateman gradient and the maximum intensity of sexual selection. Evolution 63:1673-1684.

Jones, A. G., D. E. Walker, C. Kvarnemo, K. Lindström, and J. C. Avise. 2001. How cuckoldry can decrease the opportunity for sexual selection: data and theory from a genetic parentage analysis of the sand goby, Pomatoschistus minutus. Proceedings of the National Academy of Sciences of the USA 98:9151-9156.

Kaiser, S. A., B. B. Risk, T. S. Sillett, and M. S. Webster. 2016. Data from: Ecological and social factors constrain spatial and temporal opportunities for mating in a migratory songbird. American Naturalist, Dryad Digital Repository, http://dx.doi.org/10.5061/dryad.ck0ts.

Kaiser, S. A., T. S. Sillett, B. B. Risk, and M. S. Webster. 2015. Experimental food supplementation reveals habitat-dependent male reproductive investment in a migratory bird. Proceedings of the Royal Society B 282:20142523.

Kaiser, S. A., T. S. Sillett, and M. S. Webster. 2014. Phenotypic plasticity in hormonal and behavioural responses to changes in resource conditions in a migratory songbird. Animal Behaviour 96:19-29.

Kalinowski, S. T., M. L. Taper, and T. C. Marshall. 2007. Revising how the computer program CERVUS accommodates genotyping error increases success in paternity assignment. Molecular Ecology 16: 1099-1106.

Kempenaers, B. 1993. The use of a breeding synchrony index. Ornis Scandinavica 24:84.

Komdeur, J. 2001. Mate guarding in the Seychelles warbler is energetically costly and adjusted to paternity risk. Proceedings of the Royal Society B 268:2103-2111.

Kutner, M. H., C. J. Nachtsheim, and J. Neter. 2004. Applied linear regression models. 4th ed. McGraw-Hill, New York.

Kuznetsova, A., P. B. Brockhoff, and R. H. B. Christensen. 2013. lmerTest: tests for random and fixed effects for linear mixed effect models (lmer objects of lme4 package). R package version 2-0. https://cran.r-project.org/web/packages/lmerTest/index.html.

Lack, D. 1954. The natural regulation of animal numbers. Oxford University Press, Oxford.

Magrath, M. J. L., and J. Komdeur. 2003. Is male care compromised by additional mating opportunity? Trends in Ecology and Evolution 18:424-430.

Martin, T. E. 1987. Food as a limit on breeding birds: a life-history perspective. Annual Review of Ecology and Systematics 18:453-487.

Mayer, C., and G. Pasinelli. 2013. New support for an old hypothesis: density affects extra-pair paternity. Ecology and Evolution 3:694-705.

McDonald, G. C., R. James, J. Krause, and T. Pizzari. 2013. Sexual networks: measuring sexual selection in structured, polyandrous populations. Philosophical Transactions of the Royal Society B 368:1-10. 
Miller, C. W., and E. I. Svensson. 2014. Sexual selection in complex environments. Annual Review of Entomology 59:427-445.

Møller, A. P., and T. R. Birkhead. 1993. Cuckoldry and sociality: a comparative study of birds. American Naturalist 142:118-140.

. 1994. The evolution of plumage brightness in birds is related to extrapair paternity. Evolution 48:1089-1100.

Møller, A. P., and P. Ninni. 1998. Sperm competition and sexual selection: a meta-analysis of paternity studies of birds. Behavioral Ecology and Sociobiology 43:345-358.

Nagy, L. R., and R. T. Holmes. 2005. To double-brood or not? individual variation in the reproductive effort in black-throated blue warblers (Dendroica caerulescens). Auk 122:902-914.

Newton, I. 1998. Population limitation in birds. Academic Press, San Diego, CA.

O’Brien, E. L., and R. D. Dawson. 2011. Plumage color and food availability affect male reproductive success in a socially monogamous bird. Behavioral Ecology 22:66-72.

Olsson, M., E. Wapstra, T. S. Schwartz, T. Madsen, B. Ujvari, and T. Uller. 2011. In hot pursuit: fluctuating mating system and sexual selection in sand lizards. Evolution 65:574-583.

Pedersen, M. C., P. O. Dunn, and L. A. Whittingham. 2006. Extraterritorial forays are related to a male ornamental trait in the common yellowthroat. Animal Behaviour 72:479-486.

R Development Core Team. 2015. R: a language and environment for statistical computing. Version 3.1.3. R Foundation for Statistical Computing, Vienna. http://www.R-project.org.

Reid, J. M., P. Arcese, and S. Losdat. 2014. Genetic covariance between components of male reproductive success: within-pair vs. extra-pair paternity in song sparrows. Journal of Evolutionary Biology 27:20462056.

Roberts, J. D., and P. G. Byme. 2011. Polyandry, sperm competition, and the evolution of anuran amphibians. Advances in the Study of Behavior 43:1-53.

Robinson, M. R., G. S. van Doorn, L. Gustafsson, and A. Qvarnström. 2012. Environment-dependent selection on mate choice in a natural population of birds. Ecology Letters 15:611-618.

Robinson, S. K., and R. T. Holmes. 1982. Foraging behavior of forest birds: the relationships among search tactics, diet, and habitat structure. Ecology 63:1918-1931.

Rodenhouse, N. L., T. S. Sillett, P. J. Doran, and R. T. Holmes. 2003. Multiple density-dependence mechanisms regulate a migratory bird population during the breeding season. Proceedings of the Royal Society B 270:2105-2110.

Rubenstein, D. R. 2007. Territory quality drives intraspecific patterns of extrapair paternity. Behavioral Ecology 18:1058-1064.

Ryder, T. B., R. C. Fleischer, W. G. Shriver, and P. P. Marra. 2012. The ecological-evolutionary interplay: density-dependent sexual selection in a migratory songbird. Ecology and Evolution 2:976-987.

Scelza, B. A. 2011. Female choice and extra-pair paternity in a traditional human population. Biology Letters 7:889-891.

Schlicht, L., M. Valcu, and B. Kempenaers. 2014. Spatial patterns of extra-pair paternity: beyond paternity gains and losses. Journal of Animal Ecology 83:1-14.

Schwarz, P. A., T. J. Fahey, and C. E. McCulloch. 2003. Factors controlling spatial variation of tree species abundance in a forested landscape. Ecology 84:1862-1878.

Sillett, T. S., N. L. Rodenhouse, and R. T. Holmes. 2004. Experimentally reducing neighbor density affects reproduction and behavior of a migratory songbird. Ecology 85:2467-2477.
Sinclair, A. R. E. 1989. Population regulation in animals. Pages 197241 in J. M. Cherrett, ed. Ecological concepts: the contribution of ecology to an understanding of the natural world. Blackwell Scientific, Oxford.

Soulsbury, C. D. 2010. Genetic patterns of paternity and testes size in mammals. PLoS ONE 5:e9581.

Sousa, B. F., and D. F. Westneat. 2013. Variance in mating success does not produce strong sexual selection in a polygynous songbird. Behavioral Ecology 24:1381-1389.

Stenzler, L. M., R. Fraser, and I. J. Lovette. 2004. Isolation and characterization of 12 microsatellite loci from golden-winged warblers (Vermivora chrysoptera) with broad cross-taxon utility in emberizine songbirds. Molecular Ecology Notes 4:602-604.

Stutchbury, B. J., and E. S. Morton. 1995. The effect of breeding synchrony on extra-pair mating systems in songbirds. Behaviour 132: 675-690.

Taff, C. C., C. R. Freeman-Gallant, P. O. Dunn, and L. A. Whittingham. 2013. Spatial distribution of nests constrains the strength of sexual selection in a warbler. Journal of Evolutionary Biology 26:1392-1405.

Townsend, A. K., T. S. Sillett, N. K. Lany, S. A. Kaiser, N. L. Rodenhouse, M. S. Webster, and R. T. Holmes. 2013. Warm springs, early lay dates, and double brooding in a North American migratory songbird, the black-throated blue warbler. PLoS ONE 8:e59467.

Twiss, S. D., C. Thomas, V. Poland, J. A. Graves, and P. Pomeroy. 2007. The impact of climatic variation on the opportunity for sexual selection. Biology Letters 3:12-15.

Uller, T., and M. Olsson. 2008. Multiple paternity in reptiles: patterns and processes. Molecular Ecology 17:2566-2580.

Václav, R., H. Hoi, and D. Blomqvist. 2003. Food supplementation affects extrapair paternity in house sparrows (Passer domesticus). Behavioral Ecology 14:730-735.

Wan, D., P. Chang, and J. Yin. 2013. Causes of extra-pair paternity and its inter-specific variation in socially monogamous birds. Acta Ecologica Sinica 33:158-166.

Webster, M. S., H. C. Chuang-Dobbs, and R. T. Holmes. 2001. Microsatellite identification of extrapair sires in a socially monogamous warbler. Behavioral Ecology 12:439-446.

Webster, M. S., S. Pruett-Jones, D. F. Westneat, and S. J. Arnold. 1995. Measuring the effects of pairing success, extra-pair copulations and mate quality on the opportunity for sexual selection. Evolution 49: 1147-1157.

Webster, M. S., K. A. Tarvin, E. M. Tuttle, and S. Pruett-Jones. 2007. Promiscuity drives sexual selection in a socially monogamous bird. Evolution 61:2205-2211.

Westneat, D. F. 1994. To guard mates or go forage: conflicting demands affect the paternity of male red-winged blackbirds. American Naturalist 144:343-354.

. 2006. No evidence of current sexual selection on sexually dimorphic traits in a bird with high variance in mating success. American Naturalist 167:E171-E189.

Westneat, D. F., and H. L. Mays. 2005. Tests of spatial and temporal factors influencing extra-pair paternity in red-winged blackbirds. Molecular Ecology 14:2155-2167.

Westneat, D. F., and P. W. Sherman. 1997. Density and extra-pair fertilizations in birds: a comparative analysis. Behavioral Ecology and Sociobiology 41:205-215.

Westneat, D. F., P. W. Sherman, and M. L. Morton. 1990. The ecology and evolution of extra-pair copulations in birds. Current Ornithology 7:331-370. 
Westneat, D. F., and I. R. K. Stewart. 2003. Extra-pair paternity in birds: causes, correlates, and conflict. Annual Review of Ecology and Systematics 34:365-396.

While, G. M., T. Uller, and E. Wapstra. 2011. Variation in social organization influences the opportunity for sexual selection in a social lizard. Molecular Ecology 20:844-852.

Whittingham, L. A., and P. O. Dunn. 2005. Effects of extra-pair and within-pair reproductive success on the opportunity for selection in birds. Behavioral Ecology 16:138-144.
Yezerinac, S. M., P. J. Weatherhead, and P. T. Boag. 1995. Extra-pair paternity and the opportunity for sexual selection in a socially monogamous bird (Dendroica petechia). Behavioral Ecology and Sociobiology 37:179-188.

Zuur, A. F., E. N. Ieno, N. Walker, A. A. Saveliev, and G. M. Smith. 2009. Mixed effects models and extensions in ecology with R. Springer, New York.

Associate Editor: Jeff Leips Editor: Judith L. Bronstein
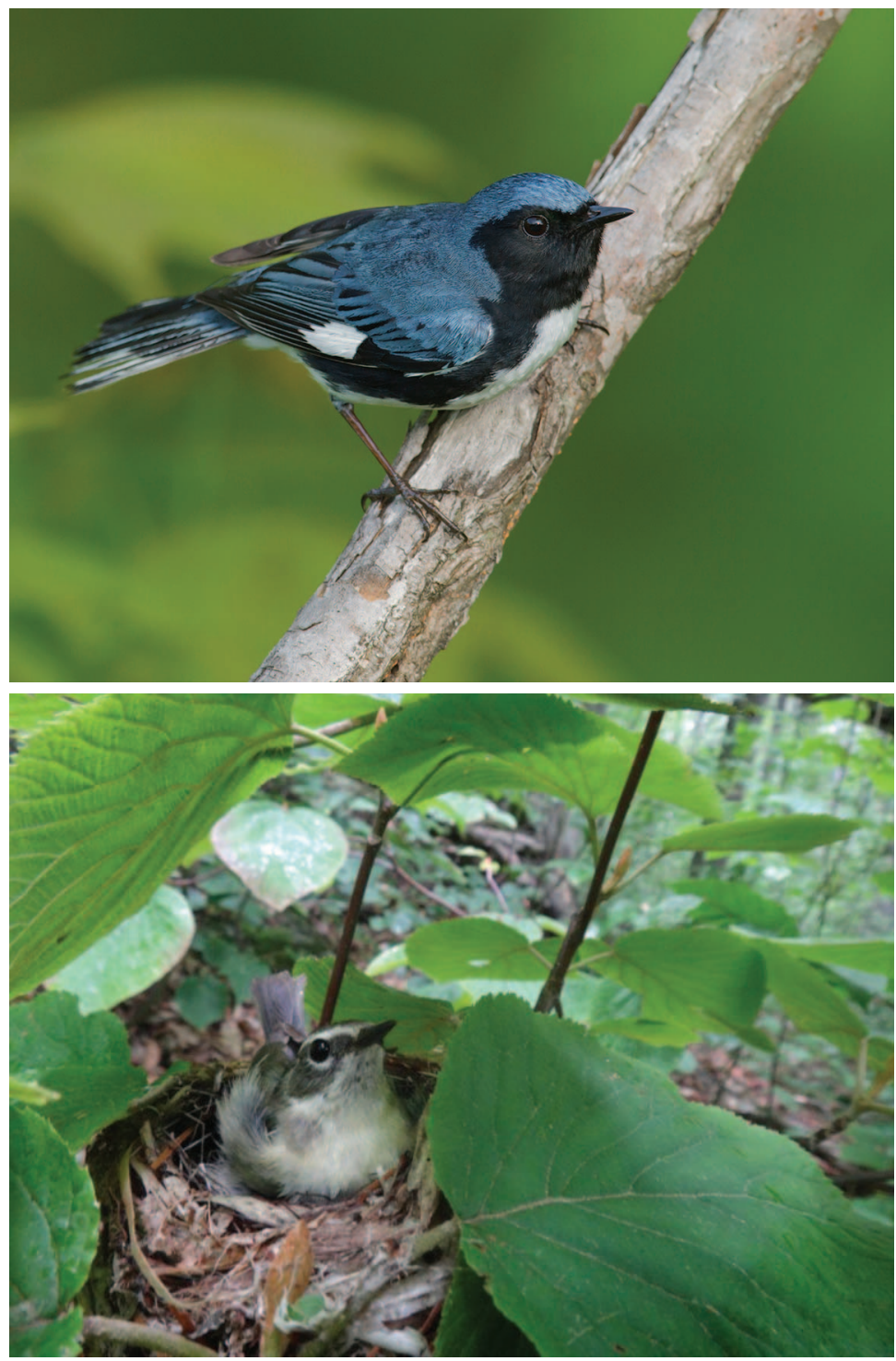

Top, male black-throated blue warbler (Setophaga caerulescens). Photo credit: Robert Royse. Bottom, female black-throated blue warbler (S. caerulescens). Photo credit: Rebecca Koch. 